\title{
Der Weichselzopf - Krankheit, Aberglaube, Charakterprofil und Erinnerungskultur
}

\section{The Elflock-Disease, Superstition, Character Profile and Remembrance Culture}

\author{
Autor \\ R. Möhn
}

Bibliografie

Dol $10.1055 / \mathrm{s}-2007-995559$

Akt Dermatol 2008; 34:

45-51 ๑ Georg Thieme

Verlag KG Stuttgart · New York ISSN 0340-2541

Korrespondenzadresse

Dr. med. Rudolf Möhn

Arzt für Hautkrankheiten und Allergologie

Philippsruher Allee 35

63454 Hanau

RMoehn@aol.com

\section{Zusammenfassung $\nabla$}

Die Ärzte früherer Jahrhunderte haben aus einer spontanen, im Verlauf der unterschiedlichsten Erkrankungen, zusätzlich mit Infektionen aller Art, mangelhafter Hygiene und Verlausung auftretenden Haarverfilzung eine Krankheit sui generis gemacht, die sog. Weichselzopfkrankheit. Das Erscheinen eines mehr oder weniger prächtigen Zopfes war zwar ein schwerer Schicksalsschlag, wurde aber andererseits häufig nicht ungern gesehen, da man glaubte, der Zopf diene der Ausleitung schlechter Körpersäfte und ein ungestörtes Wachstum führe später zur Heilung von schwerer Krankheit. Ein Abschneiden in der Blütephase war daher streng verboten und man ver- suchte sogar, sich künstlich einen Zopf zu verschaffen. Ursächlich spielten abergläubische Vorstellungen mit Zauberei und Hexerei eine große Rolle, finstere Mächte aber auch gute Geister hatten ihre Hand im Spiel. Der Weichselzopf war jedoch nicht nur ein medizinisches Thema und ein Gegenstand des Aberglaubens, sondern er diente auch zur Schärfung des Charakterprofils mit Demonstration von Überlegenheit und Machtanspruch. Das wichtigste Ziel dieses Beitrages ist es, noch eine vierte Dimension des Themas herauszuarbeiten, nämlich das überaus häufige Vorkommen in Polen nicht medizinisch, sondern als ein Stück spezifisch nationaler Erinnerungskultur zu deuten.

\section{Einleitung \\ $\nabla$}

In den letzten Jahren wurde das Thema Weichselzopf (plica polonica), das die Mediziner fast ganz Europas über Jahrhunderte in Form der so genannten Weichselzopfkrankheit beschäftigte, in „Aktuelle Dermatologie“ zweimal behandelt (Bauer, Möhn, [1,2]). Hier soll noch einmal eine zusammenfassende Betrachtung folgen, der Schwerpunkt der Arbeit aber auf der nicht medizinischen Beantwortung der rätselhaften Frage liegen, warum der Weichselzopf gerade in Polen so häufig war.

\section{Die Medizingeschichte der Weichselzopfkrankheit $\nabla$}

Wir sahen in den vorher genannten Arbeiten, dass die alten Ärzte aus einer spontanen, akzidentellen und unspezifischen Haarverfilzung und Haarverklebung, sekundär verändert durch mangelhafte Hygiene, Verlausung und Hautinfektionen aller Art eine Krankheit sui generis ge- macht haben, unter deren geräumigem Dach sich eine Fülle von Krankheitsbeschwerden körperlicher und psychischer Art subsummieren ließ.. „malum et virus hoc, non modo circa capillos, sed in venis, nervis, musculis, carne et ossibus haerens, vehementer homines cruciat et torquet, ossa interdum non solum loco movet, sed etiam in partes minutas confringit.“ Zu Deutsch: Dieses Übel und Gift sitzt nicht nur in den Haaren fest, sondern auch in den Adern, den Nerven, den Muskeln, im Fleische und in den Knochen, es quält und peinigt die Menschen heftig, es disloziert nicht nur die Knochen, sondern zerbricht sie auch in kleine Teile (Du Cange, Leben des heiligen Ladislaus) zitiert nach Schlegel [3]). Die durch finstere dämonische Mächte bewirkte und sich in mannigfacher, oft die gesamte Körperbehaarung betreffende Haarverfilzung ( $\bullet$ Abb. 1) führte zur Produktion der sog. Weichselzopfmaterie, die wir uns als ein Gemisch von Schweiß, Eiter, Krusten, Blut und Parasitenbestandteilen vorzustellen haben. Ihr Auftreten zeigte eine akute schwere, häufig genug tödliche Krankheit an, von der man, wie schon im Titel bei Schlegel [3] ersichtlich, an- 


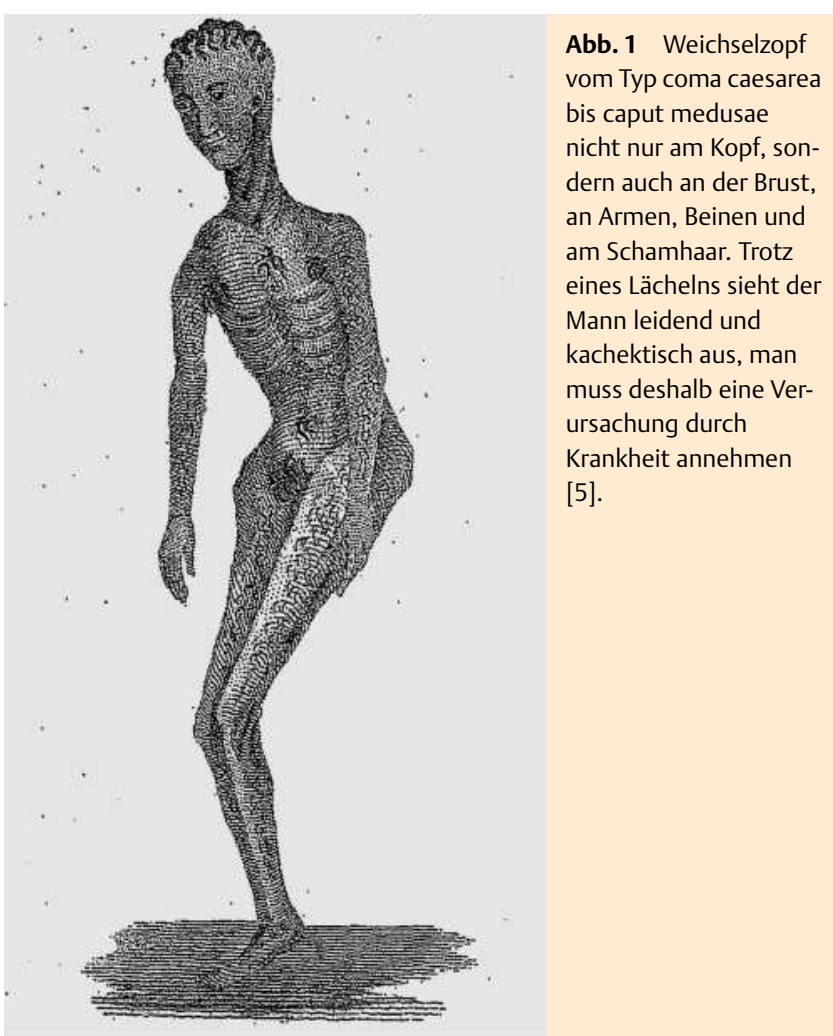

nahm, dass sie für viele Todesfälle besonders in Polen verantwortlich sei und zur Verarmung und Entvölkerung des Landes erheblich beigetragen habe. In einer weiteren Phase der Entwicklung wurde sie aber andererseits wegen der Ausleitung schlechter Säfte und der kritischen Reinigung von Kontagien und Miasmen auch als prognostisch günstiges Zeichen gesehen. Nur folgerichtig, dass ein blühender Zopf keineswegs abgeschnitten werden durfte, ja dass man sogar versuchte, sich durch artifizielle Maßnahmen, wie Pech, Wachs, Fäden und fragwürdige medikamentöse Zubereitungen, eine künstliche plica zur Therapie und Prophylaxe aller möglichen Krankheiten zu verschaffen. Sogar das Nagelorgan wurde in die Betrachtung einbezogen, und die Bezeichnung Weichselzopf auch für meist wohl durch Pilzbefall völlig destruierte Nägel verwendet ( $\bullet$ Abb. 2). Wie uns besonders die Arbeit von Bauer [1] zeigt, bereitete erst die gegen Mitte bis Ende des 19. Jahrhunderts erstarkende naturwissenschaftlich begründete Medizin der Humoralpathologie und damit auch ihrem Produkt Weichselzopf ein langsames aber sicheres Ende.

\section{Starker Einfluss des Aberglaubens \\ $\nabla$}

Der Begriff Weichselzopf soll sich nach Jacob Grimm vom polnischen Wieszczyca (die Hexe) ableiten, andere meinen, dass er von Wichtel- oder Wüchselzopf komme. Gerade im Deutschen gibt es eine Fülle von Synonymen, die alle auf Zauberei und Aberglauben hindeuten, z. B. Hexenzopf, Alpschwanz (engl. elflock), Mahrenflechte, Trudenzopf u.v.a. ähnliche Ausdrücke aber auch in vielen anderen europäischen Sprachen. Sie haben in Literatur und Sprachwissenschaft deutliche Spuren hinterlassen. So z.B. bei Erasmus Alber (1500-1553), dem Reformator Hessens: „Alp ... gestank und fauler dampf. Gewöhnlich wird von ihm erzählt, dass er bei nächtlicher weile ... die schlafenden,

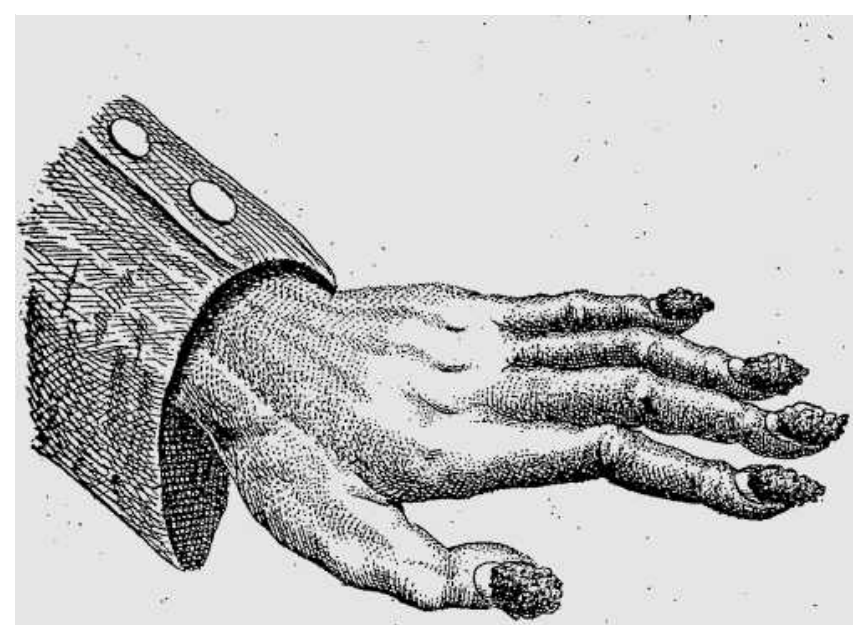

Abb. 2 Weichselzopf sämtlicher Fingernägel, offenbar Nachwachsen gesunder Nagelsubstanz proximal.

träumenden reite und drücke, zumal ihre Haare verwirre.“ Oder bei Johann Georg Wirsung (1589-1643), dem berühmten Anatomen und Erstbeschreiber des ductus pancreaticus in Arzneibuch 40: „Contorsio lautet einkrümmen, verwirren, welches wir Marflechten nennen." Und bei A. v. Droste-Hülshoff Gedichte, Band 40: „krankhafte Funken im verwirrten Haar elektrisch blitzten, und ein dunkler Mahr sich über sie ... legte." Alle diese Zitate auch bei Grimm [4]. Ein Trudenzopf war das Zeichen der keltischen priesterlichen Druiden. Ein Trudenstein ist ein von Natur durchlöcherter Stein, der in Haus oder Pferdestall aufgehängt vor Haarverwirrung bei Mensch und Tier schützt. All dies unterstreicht die wichtige Rolle, die abergläubische Vorstellungen bei dem Themenkomplex spielten. So wird immer wieder berichtet, dass jemandem der Zopf bei irgendeiner Feier von einer missgünstigen Person angehext worden sei, dass er nur unter zeremoniellen, oft durch einen Priester unterstützten Bedingungen und nur zu bestimmten Zeiten, besonders bei Neumond oder zum Osterfest abgeschnitten werden dürfe, und nach unsachgemäßer Behandlung alle möglichen Krankheiten, typischerweise oft Erblindung oder andere Augenkrankheiten aufträten. Besonders häufig wird davon nach Abschneiden eines Weichselzopfnagels berichtet. In geradezu grotesker Weise berichtet de la Fontaine (Quelle s. Leg. Abb 2, Text S. 59) von „einem ausländischen Geistlichen von 36 Jahren, der während seines 10-jährigen Aufenthaltes in Polen in vier Jahren 10-12-mal an Augenentzündungen litt. Die Erfahrung lehrte ihn endlich, dass er jedes Mal einer Augenentzündung ausgesetzt sey, wenn er sich die Nägel an den Füssen abschnitt, die Ursache einer in die Nägel übergehenden Weichselzopfmaterie wurde eingesehen, das Abschneiden wurde verboten, und Einreibungen mit Cantharidenessenz und ausleitende Mittel ergaben Heilung.“

\section{Der Weichselzopf als Ausdruck von Machtanspruch und Charakter $\nabla$}

Die abergläubischen und medizinischen Beweggründe waren aber nicht die einzigen, sich einen künstlichen Zopf zu verschaffen, sondern wie bereits in [2] dargelegt, wollten besonders Träger des Typs caput medusae ( $\bullet$ Abb. $\mathbf{3}$ ) und des keulenförmigen Typs ( Abb.4) mit ihrem ganzen Habitus ihr Charakterprofil schärfen, exklusive Überlegenheit und Machtanspruch demons- 


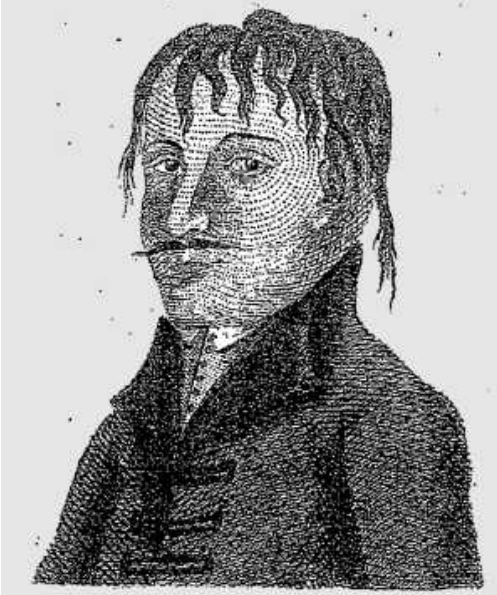

Abb. 3 Weichselzopf vom Typ coma caesarea bis caput medusae. Gesundes Aussehen des polnischen Mannes in seiner ,nationalen Haustracht", daher sicher artifiziell. Nach Schlegel [5], der von einem „endemischen“ Zopf spricht.

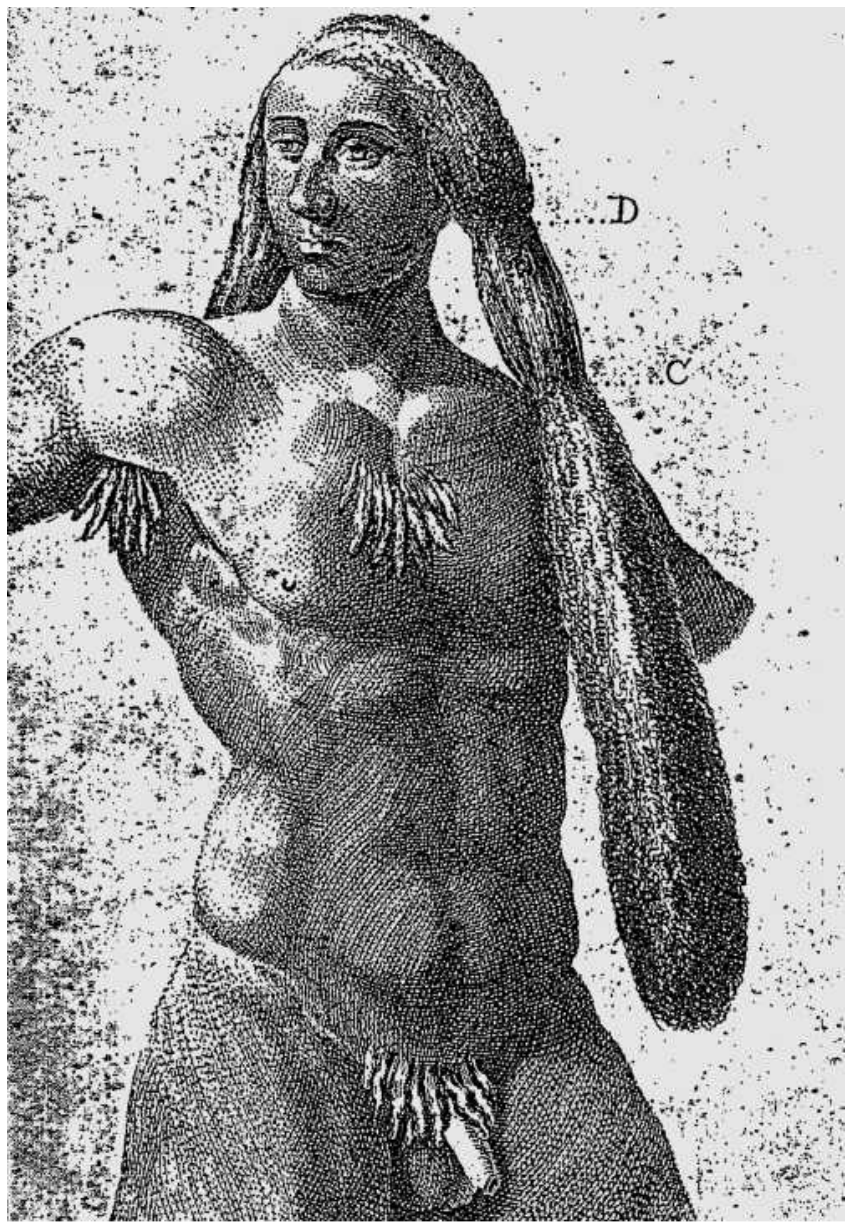

Abb. 4 Keulenförmiger Weichselzopf des Kopfes und Typ caput medusae an Achseln, Brust und Genitale. Nach de la Fontaine ( $\bullet$ Abb. 2), der dazu schreibt: „Dreyfacher oder complicierter Weichselzopf. Der ... unterste hängt an einem falschen Weichselzopf und kann bei $C$ abgeschnitten werden. Der zweite wahre nachgekommene W. sitzt noch am Kopf fest und darf so lange nicht berührt werden, bis er an neu nachgewachsenen Haaren los hängt. In diesem Fall können zwei W. auf einmal abgeschnitten werden: nemlich der unterste wahre bei $C$ und der mittlere falsche bei $D$, auch der W. unter dem Arm, Brust und Geburtsgliedern kann schon abgeschnitten werden, weil er auch schon an neuen gesunden Haaren los hängt." Der gesund aussehende junge Mann strahlt ein starkes, dandyhaftes Selbstbewusstsein und Überlegenheitsgefühl aus. Er tut etwas für sein Charakterprofil, daher sicher artifiziell.

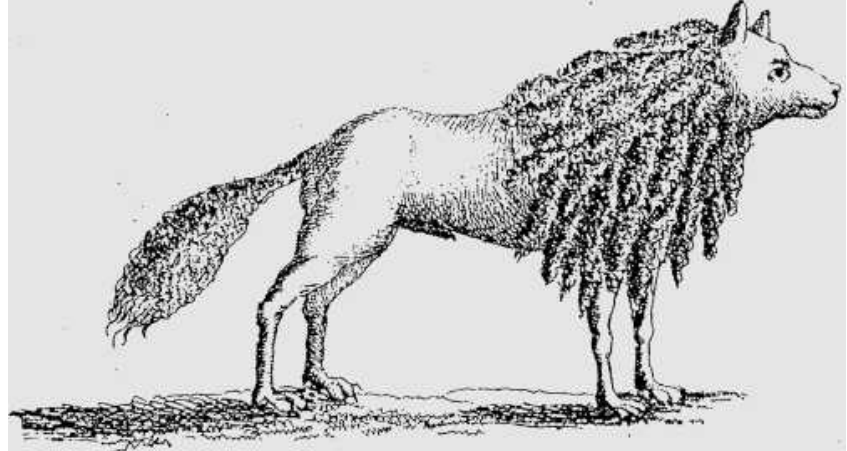

Abb. 5 Hund mit Weichselzopf an Hals und Schwanz. Er erreicht damit fast das Aussehen eines Löwen, nach de la Fontaine[16] ( $\bullet$ Abb. 2).

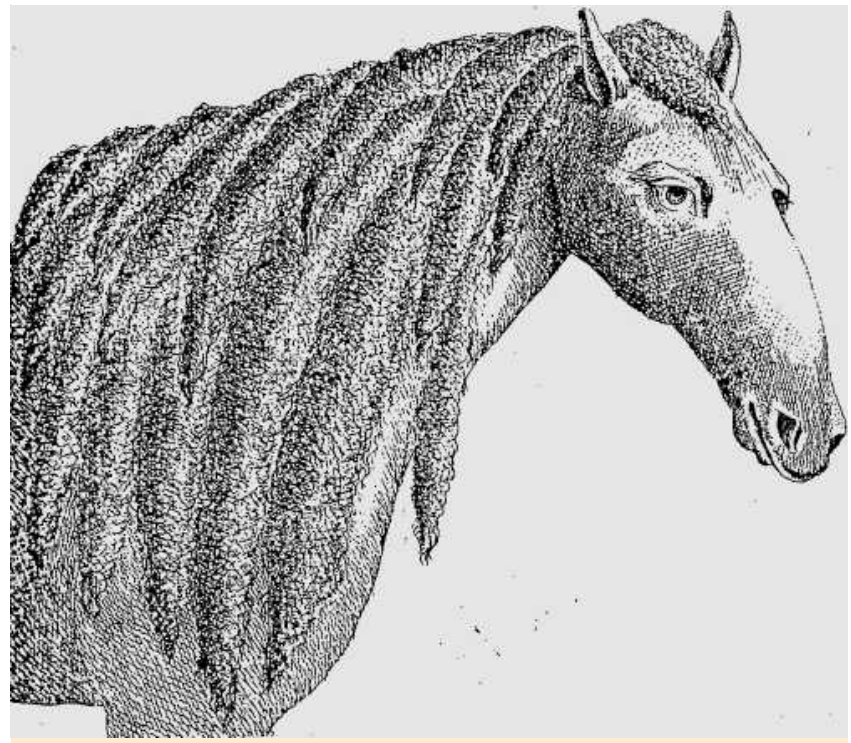

Abb. 6 Pferd mit Weichselzopfmähne. Es wird damit zu einem furchterregenden Schlachtross. Nach de la Fontaine[16] ( $\bullet$ Abb. 2).

trieren und vielleicht sogar Angst und Schrecken verbreiten. Dies zeigte sich sogar bei Tieren, die wie ihre Herren oftmals ebenso mit einem Weichselzopf herumliefen. Man beachte, wie in $\odot$ Abb. 5 aus einem einfachen Hund durch Haarverfilzung an Hals und Schwanz ein mächtiger Löwe, und wie aus einem Pferd mit Weichselzopfmähne ein gefürchtetes Schlachtross wird ( $\odot$ Abb.6). Auf die Weichselzopfmähne bezieht sich übrigens Shakespeare in „Romeo und Julia“ I,4. "Eben diese Mab verwirrt der Pferde Mähnen in der Nacht und flicht in struppges Haar die Weichselzöpfe (engl. elflock), die wiederum entwirrt auf Unglück deuten. Dies ist die Hexe, welche Mädchen drückt." Auch hier die typische Ambivalenz, der Zopf einerseits ein gefürchtetes Übel, seine Entwirrung andererseits aber problematisch, auf Unglück deutend und daher vielleicht sogar unerwünscht. Frauen konnten durch die artifiziellen Verklebungen, gleichsam als Haarsprayersatz eine entscheidende Verbesserung ihrer Haarfrisur erzielen und dadurch ihre weibliche Attraktivität steigern ( $\bullet$ Abb. 7). Sogar über den Weichselzopf bei Kindern wurde verschiedentlich berichtet, auch mit denen wollte man natürlich einen guten Eindruck machen. Die plica vom Typ plique solitaire mit Ganz- oder Teilrasur des übrigen Haares 


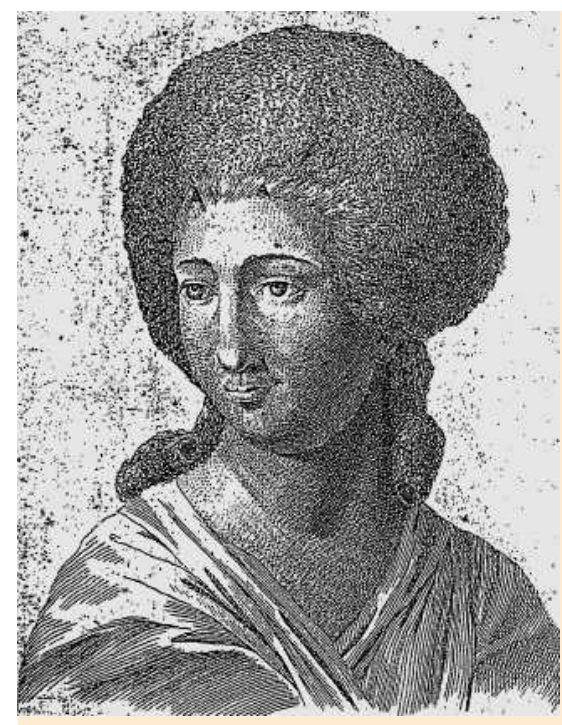

Abb. 7 Weichselzopf vom Massentyp, nach de la Fontaine [16] (॰ Abb. 2), der dazu schreibt:

„.... entweder nur bey Frauenzimmern, oder solchen Mannspersonen, deren Haupt gänzlich mit Haaren bedeckt ist. Dieser W. ist einer der unkennbarsten, indem er täglich pommadirt und gepudert, auch unter eine Haube gesteckt wird, und daher eher das Machwerk eines Friseurs, als die Wirkung einer Krankheit zu seyn scheint. Er kann bei A abgeschnitten werden, weil er vom Kopf schon absteht und an neuen gesunden Haaren hängt." Gutes Beispiel für einen artifiziellen Zopf als Kosmetikum.

( $\bullet$ Abb. 8) erinnerte an uralte bei vielen Völkern übliche Zopfrituale und Haaropfer. Ihre Träger waren oft Priester oder hatten sonst eine geistige beherrschende Funktion ( $\bullet$ Abb. 9). Im heutigen Straßenbild oder in Pop- und Rockkonzerten sind neuerdings gar nicht so selten künstliche Weichselzöpfe vom Typ caput medusae zu sehen. Der Wunsch nach exklusivem Außenseitertum liegt auf der Hand.

\section{Artefizielle Verursachung sehr häufig} $\nabla$

Man muss darum zu dem Schluss kommen, dass bei fast allen Weichselzopfformen, egal ob aus medizinischen oder anderen Gründen, artifizielle Verursachung eine besondere Rolle spielte. Diese weit über die eigentliche Medizinhistorie hinausgehenden Betrachtungen führen nach meiner Ansicht auch zur Lösung des Problems, warum der Weichselzopf gerade in Polen so besonders häufig („endemisch“ nach Schlegel [3]) gewesen ist, denn die Diskussion der in der Medizin üblichen ätiologischen Faktoren, wie landestypische Ernährung, genetische Disposition, hoher Alkoholkonsum, Wasserqualität, Bodenbeschaffenheit, Brunnenvergiftung, Infektionen aller Art u.v.a. hatten bei der Beantwortung dieser Frage zu wenig überzeugenden Ergebnissen geführt. Sie muss daher anderweitig beantwortet werden.

\section{Der Weichselzopf in Polen - ein Stiick Nationalgeschichte \\ $\nabla$}

In ganz auffallender Weise und in unbewusster Vorausschau auf eine noch unterentwickelte Völkerkunde und Kollektivpsychologie stößt man gerade auch in der umfangreichen medizinischen Literatur mehrerer Jahrhunderte wiederholt auf eine "Schlüsselgeschichte“ aus dem polnischen Mittelalter. Wir folgen hier den Ausführungen Schlegels [5], der die Wichtigkeit
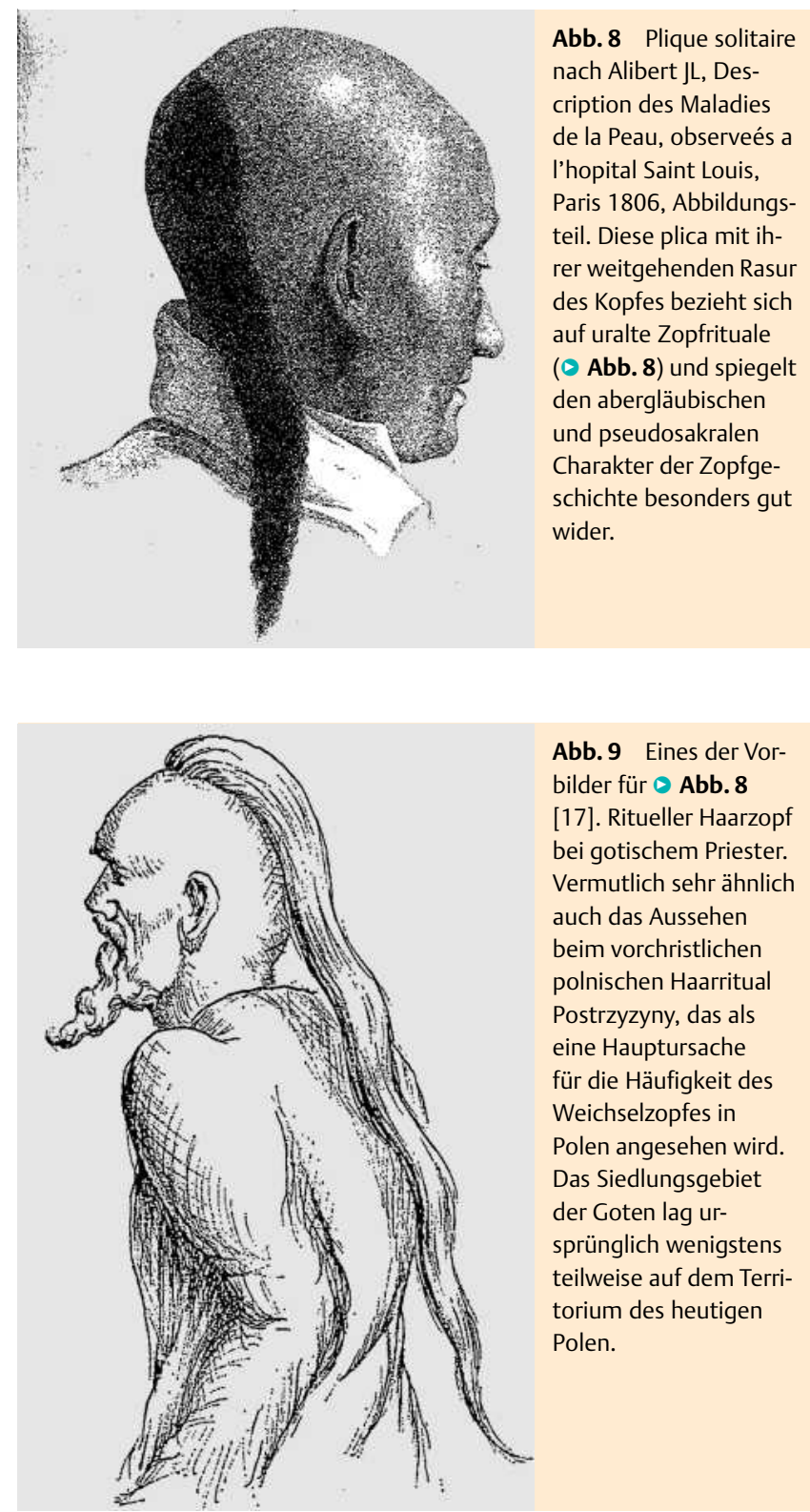

dieser Geschichte instinktiv erkannte: Nach inneren Unruhen im Lande starb 1034 der polnische König oder Herzog Mieszko II, der mit seiner Gemahlin Richeza, einer Nichte (oder Enkelin?) des deutschen Kaisers Otto III, nur einen Sohn und direkten Erben hatte, genannt Kasimir. Dieser hatte nach Wanderjahren, die ihn nach Paris, Italien und zurück nach Frankreich führten, in der berühmten Abtei Cluny das Mönchsgelübde abgelegt, durfte deswegen nicht heiraten und für eine legitime Erbfolge sorgen. Aus diesem Dilemma fand der Papst einen geschickten Ausweg. Er versprach Absolution und die Entlassung aus den mönchischen Gelübden, wenn Polen jährlich eine Summe zur Unterhaltung einer Lampe in St. Peter, den so genannten Peterspfennig (swieuto pietrze) zahle, und fortan alle Polen eine mönchische Tonsur auf dem Kopf trügen. Diese Zwangstonsur der gesamten Bevölkerung (wahrscheinlich war nur die männliche gemeint) wurde ab 1041 durchgesetzt und Kasimirs I. Königtum und einer normalen Erbfolge stand nichts mehr im Wege. 

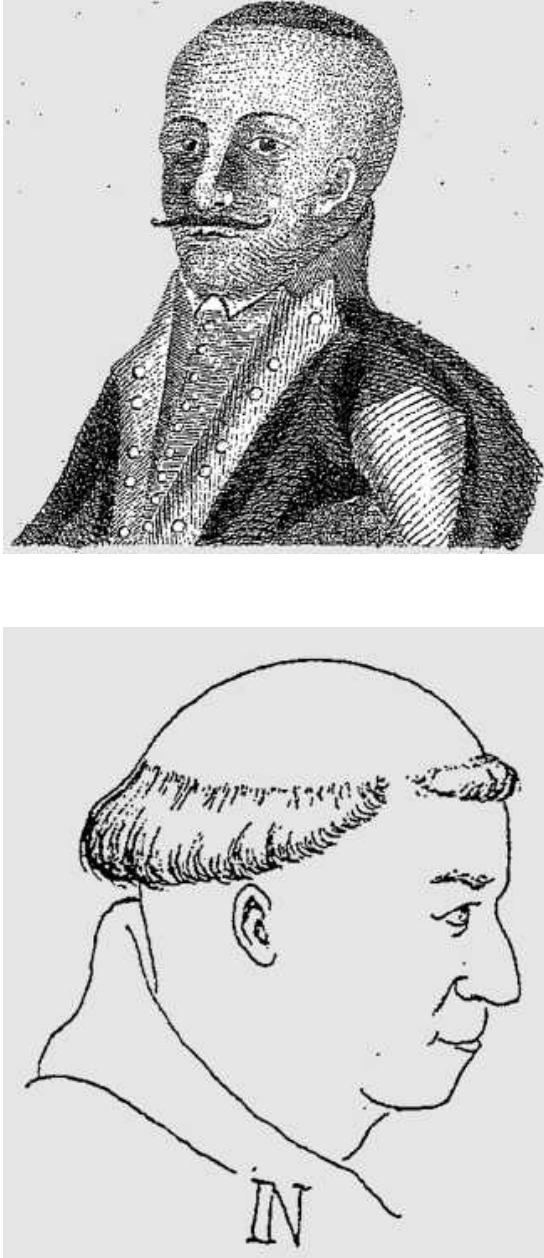

Abb. 11 Mönchische Tonsur St. Peter. Sie ist das glatte Gegenteil (eine Inversion, von normativ wird man allerdings nicht sprechen können) der polnischen Nationaltonsur. Man kann den Antagonismus zu $\odot$ Abb. 10 gut nachvollziehen.

\section{Die Bedeutung langen Haares als Ausdruck von Macht und Freiheit}

Um die Bedeutung dieser Zwangsmaßnahmen, welche die polnische Einzel- und Volksseele zutiefst gedemütigt und verletzt haben müsste, recht würdigen zu können, sollte man sich erinnern, welche Bedeutung das Tragen langen und ungeschorenen Haares bei vielen Völkern hatte. Wie beispielsweise die alttestamentliche Geschichte Samsons, oder die wallende Haartracht der Merowinger und der Völker fast ganz Europas bis ins hohe Mittelalter zeigte, waren lange Haare Garanten für Kraft, Macht und Freiheit. Die heilige Klothilde wollte ihre Enkel, weil sie niemals zur Macht gelangen und wahrscheinlich geschoren und abgeschoben würden, lieber tot als geschoren und ins Kloster gesteckt sehen und ließ sie deshalb umbringen. Überhaupt war die Devise „geschoren und ins Kloster gesteckt“ über weite Strecken des frühen Mittelalters weit verbreitet und eine grenzenlose Schande und Demütigung für den auf diese Weise unliebsam Abgeschobenen. Zudem war gerade bei den slawischen Völkern der Kopf als Sitz des Geistes und Initiator des Handelns ohnehin immer eine Problemzone gewesen. Bei dem betreffenden Papst müsste es sich um Benedikt IX (Theophylaktos), den Neffen Benedikts VIII und Johannes XIX, also einen der berüchtigten Tuskulanerpäpste, die alle skrupellose Machtmenschen waren und mit unsauberen Mitteln arbeiteten, gehandelt haben. Obwohl ihm die Zwangstonsur der Polen vielleicht zuzutrauen gewesen wäre, muss die Geschichte wohl hauptsächlich in Anlehnung an den berühmten polnischen Historiker Naruszewicz
(1733 - 1796), auf den sich Butzke [6] bezieht, dennoch als historisch unhaltbar angesehen werden, denn von einem Mönchtum des bedeutenden Königs Kasimir (der Erneuerer) ist aus den allerdings sehr spärlichen Quellen nichts bekannt geworden. Auch Seibt [7] weiß hiervon nichts zu berichten.

\section{Antagonismus von mönchischer Tonsur zu heidnischem Haarritual \\ $\nabla$}

Das ist jedoch nicht das Entscheidende. Wenn die Geschichte wahr ist, wäre sie der Ausgangspunkt für eine schwere Kollektivneurose gewesen, im Falle der Erfindung, oder nur ungenauen, sagenhaften, dafür aber durchschlagskräftigen Erinnerung, psychologisch paradoxerweise noch signifikanter, Ausdruck eines bereits vor langer Zeit stattgefundenen schweren, einem Kastrationskomplex ähnlichen, kollektiven Psychotraumas. Sie muss außerdem vor dem Hintergrund eines aus heidnischer Zeit in Polen überlieferten Haarrituals (Postrzyzyny, nach Matuszynski [8] und v. Ogonczyk-Zakrzewsky [9]) gesehen werden. Hierbei wurden dem Heranwachsenden nach Art der griechischen Theseis, des ägyptischen Jugendzopfes, des Haarrituals gotischer Priester oder der plique solitaire Aliberts $(\bullet$ Abb. 8, 9) die meisten Haare bis auf einen wallenden Zopf geschoren. Hieraus hat sich offensichtlich später die polnische Nationaltonsur mit bald längeren, bald kürzeren Haaren auf Kopfmitte bei vollständiger Rasur aller übrigen entwickelt ( $\bullet$ Abb. 10). In glattem Gegensatz dazu steht die mönchische Tonsur St. Peter, bei der die Kopfmitte geschoren ist, während ein Haarkranz bestehen bleibt. ( $\bullet$ Abb. 11). Wenn also in dem sowieso nur schwer zu christianisierenden Land Polen immer mehr Männer mit der mönchischen Tonsur herumliefen, muss das zu einem unheilvollen Antagonismus, zu einem unlösbaren Konflikt zwischen überliefertem Haarritual und mönchischer Tonsur geführt haben. Die Erinnerung an Postrzyzyny wurde durch das Christentum zwar bekämpft, aber in veränderter Form von Zopf und Filz auf fatale Weise aufrechterhalten. Es kam zu einer Aktivierung des polnischen kulturellen Gedächtnisses, in dem auch die Geschichte von König Kasimir hartnäckig persistierte.

\section{Kollektives Psychotrauma nach Freud} $\nabla$

Die weiteren erläuternden Ausführungen verdanke ich ganz wesentlich den Arbeiten über Erinnerungskultur und kulturelles Gedächtnis von Jan Asmann [10,11],der in seinen Werken ausführlich dieses relativ junge wissenschaftliche Fach behandelt, verschiedene Denkmodelle präsentiert [10] und sich u.a. mit Siegmund Freud beschäftigt, dessen bleibendes Verdienst es ist, die Erkenntnisse aus der Individualpsychologie auf die Psychodynamik von Gruppen, Völkern oder gar der ganzen Menschheit angewandt zu haben. In „Totem und Tabu“ sowie „Der Mann Moses“ versucht er, gewisse Gesetzmäßigkeiten herauszuarbeiten, die stichwortartig so formuliert werden können: Schweres psychisches Trauma (in unserem Fall die Zwangstonsur) - Abwehr Latenz (wie bei einem körperlichen Trauma, bei dem die Schmerzen oft auch erst später kommen) - Ausbruch der neurotischen Erkrankung, (als die der Weichselzopf aufgefasst werden müsste). An anderer Stelle formuliert er so: „... Wiederherstellung des Vergangenen, Wiederkehr des Vergessenen nach langen Intervallen ... es ist besonderer Hervorhebung wert, dass jedes aus der Vergessenheit wiederkehrende Stück sich mit be- 


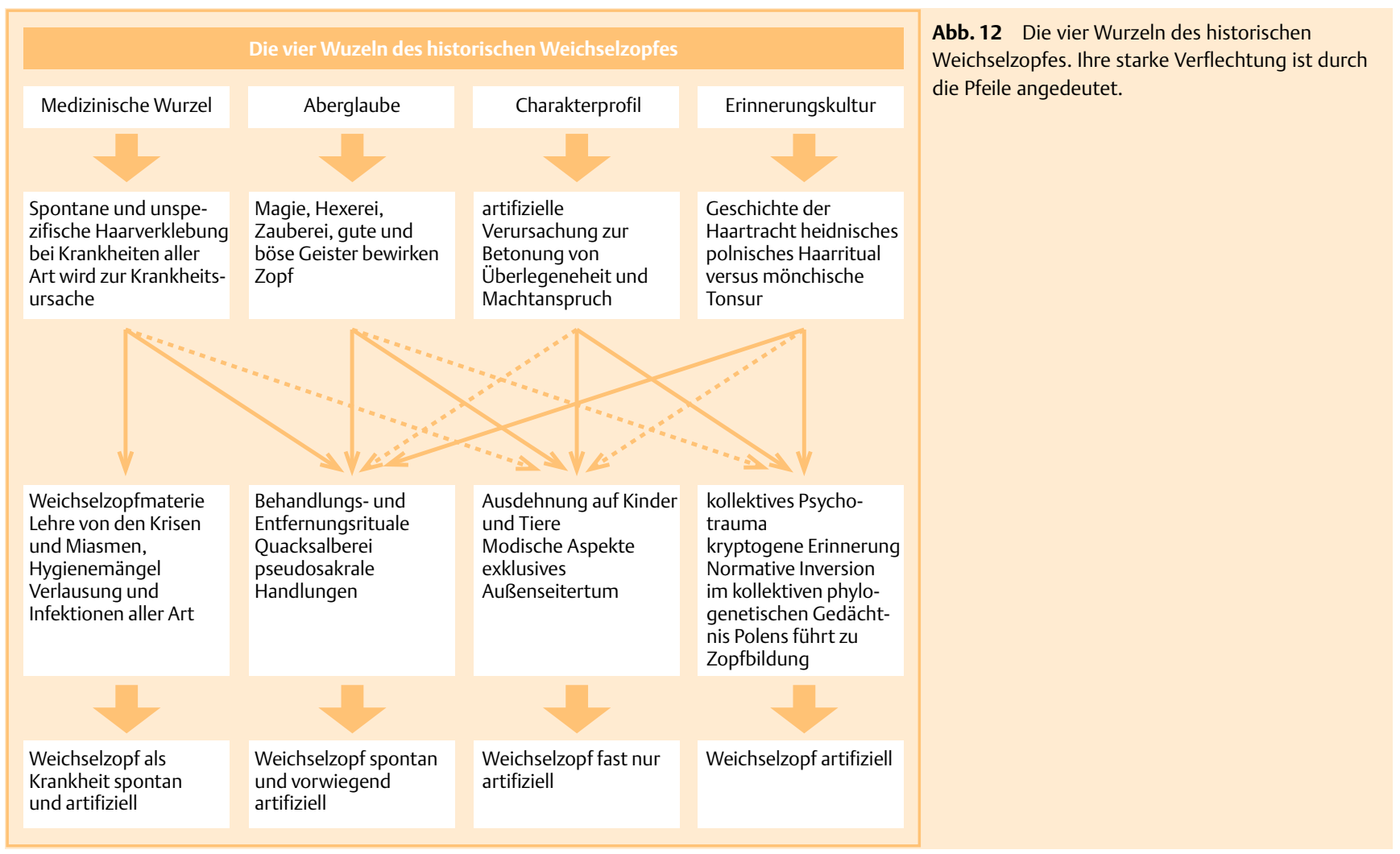

sonderer Macht durchsetzt, einen unvergleichlich starken Einfluss auf die Menschenmassen übt und einen unwiderstehlichen Anspruch auf Wahrheit erhebt, gegen den logischer Anspruch machtlos bleibt" [12]. Ich hoffe, der Leser versteht die Analogie, die darin besteht, dass der polnische Weichselzopf als stark veränderte, neurotische Wiederkehr des nur scheinbar vergessenen polnischen Haarrituals Postrzyzyny aufzufassen wäre. Die Latenz hat in unserem Falle mehrere Jahrhunderte gedauert, nämlich vom 11. bis ins 16. Jahrhundert als der berühmte StarnigelBrief vom 31.10.1599 [13], in dem die Professoren der Universität Zamosc ihre Kollegen in Padua wegen der neuen Erkrankung plica polonica um Rat fragen, ihren Ausbruch in Polen für etwa die Mitte des 16. Jahrhunderts angibt. Im Gedächtnis der Völker ist das aber keine lange Zeit!

\section{Die psychische Krypta \\ $\nabla$}

Ein zweites Denkmodell findet sich bei Jacques Derrida et al. [14], der den Begriff der „Krypta“ in die Psychologie eingeführt hat. Diese ist gleichsam ein „durch Einverleibung in das Ich eingeschlossener Fremdkörper“. Nimmt man an, dass das vorchristliche polnische Postrzyzyny trotz oder gerade wegen der Bekämpfung durch das Christentum nie ganz vergessen wurde, sondern im Gegenteil durch die Jahrhunderte, wenn auch in der stark veränderten, trotzigen Form der Haarverfilzung, eisern bewahrt wurde, kann man auch von einer psychischen „Kryptisierung“ einer sorgsam verborgenen Erinnerung sprechen. Nach Derrida ist eine solche Krypta ein „falsches oder artefizielles Unbewußtes“, ,placiert wie eine Prothese“, „ein Pfropfen im Herzen eines Organes“, „ein gespaltenes Ich“, „ein verschanztes Fort“, und in Anlehnung aber auch Widerspruch zu Siegmund Freud „eine falsche Wiederkehr des Verdrängten“, „eine falsche Bilanz“. Auch diese Begriffe wären auf den Weichselzopf als starke
Variation des ursprünglichen Postrzyzyny, das wie eine Krypta aus heidnischer Zeit in der nationalen polnischen Seele verborgen liegt, sehr gut anwendbar und könnten die hartnäckige Persistenz des Phänomens, nach Jahrhunderten der Latenz gut erklären. Das immer wieder beschriebene Mitwirken von christlichen Geistlichen bei der zeremoniellen Beseitigung alter Zöpfe und heidnischer Relikte aus alter Zeit fände hier mühelos eine Erklärung. Die „Krypta“ muss endlich zum Einsturz gebracht werden! Wenn man sich zudem überlegt, dass der „Gwozdiec“, mit dem die Polen die eigentliche Weichselzopfkrankheit bezeichnen, sozusagen der innere Kern des Leidens, der „Pfahl im Fleisch“" (Gwozd polnisch der Nagel, Pfahl) auch im somatischen Bereich kryptisch (in venis, nervis, musculis carne etc. s. 3.) tief im Körperinneren verborgen liegt, dass er durch kritische Absonderungen heraus will, dass sich ein „Koltun“, polnisch der äußerliche Zopf oder Phänotyp, unter Begleitung vieler Krankheiten bilden muss, um die Spannung aufzulösen, handelt es sich um eine erstaunliche psychische und somatische Analogie.

\section{Die Plica als spezifisch polnisch-nationale Erinnerungskultur $\nabla$}

Spricht Freud von Neurosen und kollektiven Psychotraumen, wandelt sich das bei Assmann [11] in einem dritten Denkmodell mehr in die Begriffe kulturelles Gedächtnis und Erinnerungskultur. Es gibt demnach nicht nur ein individuelles ontogenetisches Gedächtnis, sondern auch ein phylogenetisches, z. B. ein ganzes Volk betreffendes kollektives, kulturelles Gedächtnis, dessen Grundlagen u.a. auf einer mythischen Urgeschichte oder einem Ereignis in absoluter Vergangenheit, auf zeremoniellen Gemeinsamkeiten in hoher Geformtheit, Festen, zeremoniellen Kommunikationen, weiter in festen Objektivationen und traditionellen symbolischen Handlungen beruhen, die durch besonders quali- 
fizierte Handlungsträger, z. B. Priester, Schamanen und Medizinmänner bewirkt werden ([11], S. 56). Ich denke, dass die Analogien zum Weichselzopf in Polen auf der Hand liegen. Die mythische Urgeschichte in absoluter Vergangenheit liegt in Form von Postrzyzyny und der Geschichte von König Kasimir vor. Die feste Objektivation ist der monströse verfilzte Weichselzopf, der als mnemotechnisch bedingte vollkommene normative Inversion des alten polnischen Haarrituals Postrzyzyny aufgefasst werden muss, die symbolischen Handlungen und zeremoniellen Kommunikationen sind das Abschneiden des Zopfes speziell zum Osterfest, die Handlungsträger die Priester, welche dieses Geschehen in Polen halb christlich gläubig, halb abergläubisch überwacht haben. Im kollektiven Gedächtnis spielt das Phänomen der normativen Inversion eine große Rolle, wie schon Jacob Grimm, ohne allerdings diesen Terminus zu gebrauchen, vermutet hat, und wie sie sich sogar in der Weichselzopfterminologie widerspiegelt. Denn er nimmt an, dass die hässlichen und bösen Hexen die normative Inversion lieblicher germanischer Gottheiten sind, besonders der Frau Holle, Hulda oder Holda. So heißt der o.g. Hexenzopf entsprechend nämlich auch Höllenzopf, Hollenzopf oder auch Hollezaul und Hollezopp. Normativ invers übrigens auch schon rein sprachlich der böse Alp (Elb) versus liebliche Elfe.

Erinnerungskultur, Aberglaube und Charakterprofilierung werden in gleichsam säkularisierter Form später durch Krankheiten, Medizingeschichte und Ärzte überlagert, die das zunächst nur kulturgeschichtliche Phänomen der Plica zu einem medizinischen Problem gemacht haben, dabei aber selbst mit Tradition und Aberglauben zu kämpfen hatten, oft genug zu zweifelhaften Heilmethoden, Quacksalberei und außermedizinischen Vorstellungen Zuflucht nehmen mussten und so in die vom heutigen Standpunkt aus gesehen dubiose Rolle von Schamanen und Medizinmännern schlüpften.

Wir sind nach diesen Ausführungen nun in der Lage, vier verschiedene Wurzeln des Weichselzopfproblems, die wie der Zopf selbst stark ineinander verflochten sind, darzustellen und tun dies am besten in Form einer Grafik, die zugleich eine optisch einprägsame Zusammenfassung sein möge ( $\bullet$ Abb. 12).

\section{Abstract}

\section{The Elflock-Disease, Superstition, Character Profile and Remembrance Culture \\ $\nabla$}

The phenomenon of matted hair which appeared spontaneously in the course of different illnesses combined with all sorts of infections, poor hygiene and infestation with lice, namely the socalled elflock disease, was made a disease „sui generis“ by the doctors of earlier centuries. The appearance of a more or less gorgeous elflock was on the one hand a severe blow of fate, on the other hand it was often not altogether undesirable, because it was believed that the elflock provided an exit for bad juices out of the body and an unhampered growth would eventually lead to the cure of a serious illness. Cutting off the elflock in its prime was therefore strictly forbidden and people even tried to acquire an artificial one. Superstitious attitudes of sorcery and witchcraft played a fundamental role, dark forces as well as good spirits were involved. However the elflock was not only an issue of medicine or superstitition, but it was also a means of sharpening the character profile with the demonstration of superiority and claim to power. The aim of this essay is primarily to emphasize a fourth dimension of the topic, namely to interpret the widespread appearance in Poland not as a medical phenomenon but as an element of a specifically national remembrance culture.

\section{Literatur}

1 Bauer AW. Der Weichselzopf in medizinhistorischer Perspektive. Eigenständige Hautkrankheit oder mythologisches Konstrukt. Akt Dermatol 2004; 30: $218-222$

2 Möhn R. Das Medusenhaupt in Mythos und Medizin - Versuch einer ganzheitlichen Betrachtung. Akt Dermatol 2007; 33: 388 - 393

3 Schlegel JFA. Du Cange. Leben des heiligen Ladislaus. Über die Ursachen des Weichselzopfes der Menschen und Thiere, die Mittel denselben zu heilen und in kurzem auszurotten (und dem dadurch entvölkerten Polen seinen ehemaligen blühenden Wohlstand wieder zu verschaffen). Jena: bey Johann Christian Gottfried Gopferdt, 1806: 289

4 Grimm J, Grimm W. Deutsches Wörterbuch. München: Deutscher Taschenbuchverlag, 1984

5 Schlegel JFA. Über die Ursachen des Weichselzopfes der Menschen und Thiere, die Mittel denselben zu heilen und in kurzem auszurotten (und dem dadurch entvölkerten Polen seinen ehemaligen blühenden Wohlstand wieder zu verschaffen). Jena: bey Johann Christian Gottfried Gopferdt, 1806: 105

6 Butzke EL. Denkschrift über den Weichselzopf. Ein Beitrag zur Begründung einer rationellen Pathologie und Therapie desselben. Thorn: Commissionsverlag von Ernst Lambeck, 1858: 186

7 Seibt F. Polen von der Jahrtausendwende bis 1444. In: Handbuch der europäischen Geschichte (HEG), Band 2, Europa im Hoch- und Spätmittelalter. Stuttgart: Klett-Cotta, 1987

8 Matuszynski J. Über die Natur und Behandlung des Weichselzopfes. Tübingen: Inaug. Diss. bei C. F. Osiander, 1834: 5

9 Ogonczyk-Zakrzewski MF Ritter von. Medizinisch-literärische Geschichte des Weichselzopfes, ein Versuch. Wien: MechitaristenCongregations-Buchhandlung, 1830: 10

10 Assmann J. Moses der Ägypter. Entzifferung einer Gedächtnisspur. Frankfurt am Main: Fischer, 2001

11 Assmann J. Das kulturelle Gedächtnis: Schrift, Erinnerung und politische Identität in frühen Hochkulturen. München: C. H. Beck, 1999

12 Freud S. Der Mann Moses und die monotheistische Religion. Schriften über die Religion. Frankfurt am Main: Fischer, 1975: 91 und 87

13 Starnigel L. Epistula Laurentii Starnigeli. Academiae Zamoscensis Rector Eloquentiae Profess. Ad Acad. Patae Prof. Medicos, data 1599, die ult. Octobris

14 Derrida J. Das Verbarium des Wolfsmannes. In: Abraham N, Torok MKryptonymie. Frankfurt: Ullstein, 1979: 8, 33 und 63

15 Grimm J. Deutsche Mythologie von 1835. (Nachdruck) Wiesbaden: Fourier, 2003: 10

16 de la Fontaine FL. Chirurgisch-medizinische Abhandlungen Polen betreffend.Über den Weichselzopf, 1. Abhandlung Breslau und Leipzig: bey W. G. Korn, 1792; 8 ov

17 Corson $R$. Fashions in Hair, the first five thousand years. London: Peter Owen, 1965: (Abbildungsteil) 